\title{
General testing method for refractive surfaces based on reverse Hartmann test
}

Ping Xu, Daodang Wang, Zhidong Gong, Zhongmin Xie, Rongguang Liang, et al.

Ping Xu, Daodang Wang, Zhidong Gong, Zhongmin Xie, Rongguang Liang, Ming Kong, Jun Zhao, Linhai Mo, Shuhui Mo, "General testing method for refractive surfaces based on reverse Hartmann test," Proc. SPIE 10373, Applied Optical Metrology II, 103730J (23 August 2017); doi: $10.1117 / 12.2273189$

Event: SPIE Optical Engineering + Applications, 2017, San Diego, California, United States 


\title{
General testing method for refractive surfaces based on reverse Hartmann test
}

\author{
Ping Xu $\mathrm{u}^{\mathrm{a}}$, Daodang Wang ${ }^{*}$, Zhidong Gong ${ }^{\mathrm{a}}$, Zhongmin Xie ${ }^{\mathrm{a}}$, Rongguang Liang ${ }^{\dagger \mathrm{b}}$, Ming Kong ${ }^{\mathrm{a}}$, Jun \\ $\mathrm{Zhao}^{\mathrm{a}}$, Linhai $\mathrm{Mo}^{\mathrm{c}}$, Shuhui $\mathrm{Mo}^{\mathrm{c}}$ \\ ${ }^{a}$ College of Metrology and Measurement Engineering, China Jiliang University, Hangzhou 310018, \\ China; ${ }^{b}$ College of Optical Sciences, University of Arizona, Tucson, Arizona 85721, USA; ${ }^{\mathrm{c}}$ Volkslift \\ (China) Company Limited, Huzhou 313009, China
}

\begin{abstract}
The testing technique with high dynamic range is required to meet the measurement of refractive wavefront with large distortion from test refractive surface. A general deflectometric method based on reverse Hartmann test is proposed to test refractive surfaces. Ray tracing of the modeled testing system is performed to reconstruct the refractive wavefront from test surface, in which computer-aided optimization of system geometry is performed to calibrate the geometrical error. For the refractive wavefront error with RMS $255 \mu \mathrm{m}$, the testing precision better than $0.5 \mu \mathrm{m}$ is achieved.
\end{abstract}

Keywords: refractive surface testing; reverse Hartmann test; ray tracing; total internal reflection

\section{INTRODUCTION}

With the rapid development of optical fabrication, the refractive surface has been widely applied in various industrial products and systems, including the imaging optics, illuminating optics, transmission glasses and transparent bottles. It places high requirement on the performance of refractive surfaces, especially in the case of imaging optics [1]. The testing of refractive wavefront from refractive surface enables quantitative evaluation of refractive surface $[2,3]$, providing a feasible way for the quality improvement of industrial products [4].

The interferometers, such as Fizeau interferometer, Twyman-Green interferometer and point-diffraction interferometer, provide a feasible and noncontact way to test the wavefront from refractive surfaces with ultra-high accuracy in the order of nanometers [5-7]. However, the dynamic range of the interferometric testing is quite small, and it can only measure the surface departure within a few wavelengths from reference shape $[8,9]$. Specifically designed null optics and compensation optics are generally required in the testing of complex surfaces with large aberration, making the testing system very costly and inflexible [10-12]. Besides, the interferometry has high requirement on the calibration of testing system [13], which determines its achievable accuracy. The slope testing methods such as the Ronchi test and Hartmann test provide slope information of surfaces under test with high dynamic range [14]. However, it is difficult to measure convex surfaces or even the flat ones due to the limitation of testing system configuration.

The deflectometric testing method based on reverse Hartmann test is a slope measuring technique which enables the refractive surfaces testing with the characters of full-field and high dynamic range [15]. According to the measured surface slope (derivative of surface sag), the surface error can be obtained by integration method. The deflectometric system is inexpensive and simple in configuration, generally consisting of a LCD projection screen and a camera sampling the surface [16]. Besides, it is highly flexible because no null optics and compensating optics are required [17]. It provides a simple, low-cost and feasible method for the testing of large quantity of industrial refractive surfaces. The application of deflectometry are extremely diverse and wide, and the performance of deflectometry has been successfully demonstrated in the testing of astronomy telescope mirrors, spherical mirrors, aspheric mirrors and precision X-ray mirrors [18-20].

\footnotetext{
*wangdaodang@sina.com; phone 86-571-86914563

† rliang@optics.arizona.edu; phone 1-520-621-4995
}

Applied Optical Metrology II, edited by Erik Novak, James D. Trolinger, Proc. of SPIE Vol. 10373, 103730J @ 2017 SPIE · CCC code: 0277-786X/17/\$18 · doi: 10.1117/12.2273189 
In this paper, a deflectometric method is presented to achieve the accurate testing of refractive surfaces, such as lenses, curved surfaces and glass bottles based on configuration of reverse Hartmann test system. With the obtained surface data, ray tracing is carried out to realize the "null" testing of surfaces in modeled testing system based on the calibrated geometry. In addition, a mathematical model is presented to analyze the removal of the total internal reflection (TIR). After data removal of TIR, accurate testing of refractive surfaces can be achieved. Section 2 presents the principle of the deflectometry based on the reverse Hartmann test for refractive surfaces testing and the principle of TIR data removal in the testing of curved surfaces. In Sections 3 and 4, numerical simulation result and experimental result are given to demonstrate the validation and feasibility of the proposed method. In addition, some conclusions are drawn in Section 5.

\section{PRINCIPLE OF REVERSE HARTMANN TEST AND TOTAL INTERNAL REFLECTION DATA REMOVAL}

\subsection{Reverse Hartmann test}

Figure 1(a) shows the schematic diagram of reverse Hartmann test system for reflective surface testing. The flat panel display serves as the illumination screen and the image of the surface under test is captured by the CCD camera with a finite size of aperture. If a single pixel is lit up on the display, there will be a bright region corresponding to the areas of the surface on the image of the test surface. According to the deflectometric system for reflective surface testing in Fig. 1(a), the angle of incident ray is equal to the angle of the reflected ray, and the angular of these two rays is normal to the reflection surface at the bright area based on the law of reflection. The proposed deflectometric system for refractive surface testing is based on the configuration of reverse Hartmann test, as is shown in Fig. 1(b), which is similar with that in Fig. 1(a). According to Fig. 1(b), there is a definite relationship between the angle of the incident ray, the corresponding refracted ray and the slope of the surface based on the law of refraction. Thus, the correspondence of incident ray and the reflected/refracted ray is uniquely defined by the illumination screen pixel position, reflecting/refracting region in the surface and center of the camera aperture. Based on the triangulation, slope of the surface under test can be determined, from which the surface data can be obtained.

(a)

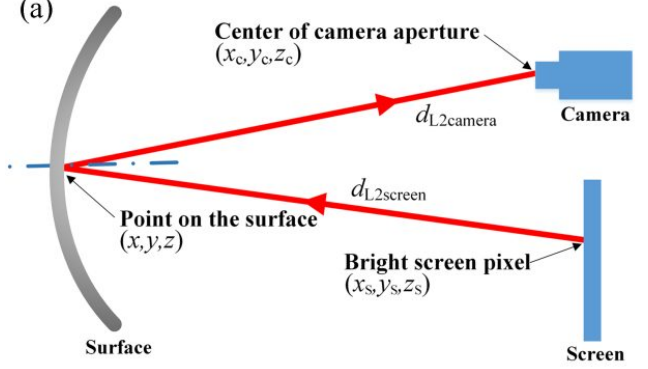

(b)

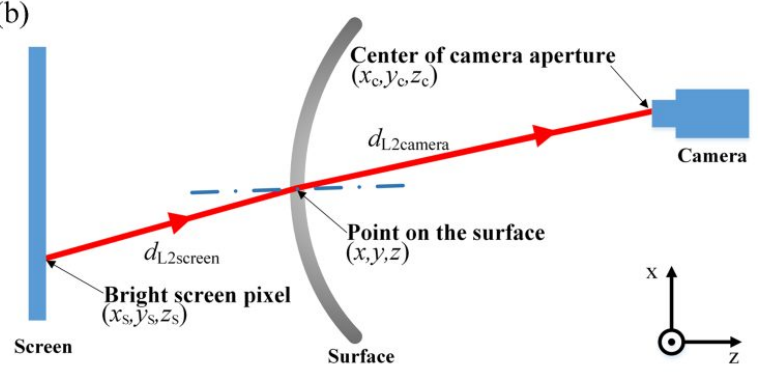

Fig. 1. Schematic diagram of reverse Hartmann test system. (a) Model for reflective surface testing, and (b) model for refractive surface testing.

By phase shifting the sinusoidal fringes on illumination screen, the unique correspondence between illumination screen pixel and the reflection region on the refractive surface is determined. The surface error, that is the surface departure from its nominal shape, can be measured by ray tracing the testing system model, in which the camera is modeled as an ideal point source sending light to the test surface in ideal shape and the LCD projection screen modeled as an image plane to capture the rays reflected/refracted from the surface. The ideal spot distribution $\left(x_{\text {ideal }}, y_{\text {ideal }}\right)$ can be obtained by ray tracing the modeled system, and the spot distribution $\left(x_{\text {actual }}, y_{\text {actual }}\right)$ in the experiment is calculated from phase shifting method. Based on transverse ray aberration model [21], the system wavefront aberration can be estimated by dividing the spot coordinate differences $(\Delta x, \Delta y)$ with the measured distance $d_{\mathrm{L} 2 \mathrm{screen}}$ between the test surface and the illumination screen, 


$$
\left\{\begin{array}{l}
\frac{\partial W(x, y)}{\partial x}=-\frac{\Delta x}{d_{\mathrm{L} 2 \text { screen }}}, \Delta x=x_{\text {ideal }}-x_{\text {actual }} \\
\frac{\partial W(x, y)}{\partial y}=-\frac{\Delta y}{d_{\mathrm{L} 2 \text { screen }}}, \Delta y=y_{\text {ideal }}-y_{\text {actual }}
\end{array},\right.
$$

where $W(x, y)$ is the wavefront aberration, $(x, y)$ is the exit pupil coordination; $d_{\mathrm{L} 2 \text { screen }}$ is the distance from the surface under test to LCD projection screen, $\Delta x$ and $\Delta y$ are the coordinate differences between spot distribution $\left(x_{\text {ideal }}, y_{\text {ideal }}\right)$ by ray tracing and measured spot distribution $\left(x_{\text {actual }}, y_{\text {actual }}\right)$. With the integration of slope difference, the test surface error can be measured.

\subsection{Analysis of TIR data removal}

In the testing of refractive surfaces such as curved glasses and transparent bottles, TIR could exist in the edge part of the surfaces, where the rays travel through the refractive surfaces and back to air, as is shown in Fig. 2(a). TIR could introduce significant error in the testing result, and the removal of TIR data is required to achieve accurate testing. A mathematical model can be set up to analyze the correspondence between the region of the surface without TIR and the parameters of testing system. Figure 2(b) gives the detail of critical ray direction on the surface. From Fig. 2(b), the critical angle $C$ of TIR can be obtained by

$$
C=\arcsin \frac{1}{n},
$$

where $n$ is the refractive index. Define the angle $\theta$ between Lines AO (connecting the point of incident on the surface and curvature center) and $\mathrm{BO}$ (connecting the point of ejection on the surface and curvature center) in Fig. 2(b), and we have the relationship

$$
\theta=C-\arcsin \frac{r}{R \cdot n}
$$

where $r$ and $R$ are the curvature radius of the back and front surfaces on the test object (such as the curved surface with certain thickness), respectively.
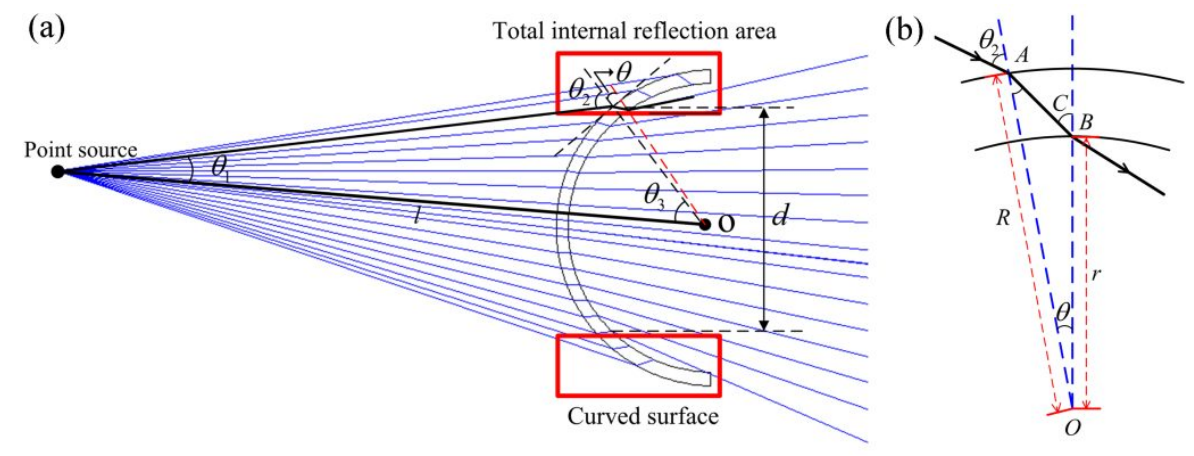

Fig. 2. Total internal reflection model. (a) Model for TIR analysis, and (b) local enlarged detail.

According to the triangulation of the TIR model in Fig. 2(a) and the critical condition in Eq. (2), the incident angle $\theta_{2}$ on refractive surface and angle $\theta_{1}$ between the incident ray and optical axis should satisfy the relationship

$$
\left\{\begin{array}{l}
\theta_{1}=\arcsin \frac{n \cdot R \cdot \sin (C-\theta)}{l} \\
\theta_{2}=\arcsin [n \cdot \sin (C-\theta)]
\end{array},\right.
$$


where $l$ is the distance between light source and curvature center. Therefore, the aperture $d$ of the region without TIR data on the surface can be calculated from

$$
\left\{\begin{array}{l}
\theta_{3}=\theta_{2}-\theta_{1} \\
d=2 R \cdot \sin \theta_{3}
\end{array} .\right.
$$

The aperture $d$ can be obtained according to parameter of the test object and testing system.

\section{NUMERICAL SIMULATION RESULTS}

\subsection{Simulation results of deflectometric testing of refractive surface}

To demonstrate the feasibility of the proposed deflectometric method for refractive surface testing based on reverse Hartmann test, the numerical simulation is carried out, in which a flat glass with $1 \mathrm{~mm}$ thickness and 1.47 refractive index is tested based on ray tracing method. A testing system, whose configuration is shown in Fig. 1(b), has been set up in Zemax for ray tracing, where the camera acts as an ideal point source and the LCD projection screen as a capture screen. The distance $d_{\mathrm{L} 2 \mathrm{camera}}$ between the point source and test surface is $183 \mathrm{~mm}$, and the distance $d_{\mathrm{L} 2 \text { screen }}$ between the test surface and screen is $153 \mathrm{~mm}$. The actual surface error of test flat surfaces are shown in Figs. 3(a) and 3(d), in which the root-mean-squares (RMS) values of surface errors are $2.9767 \mu \mathrm{m}$ and $255.0835 \mu \mathrm{m}$, respectively.
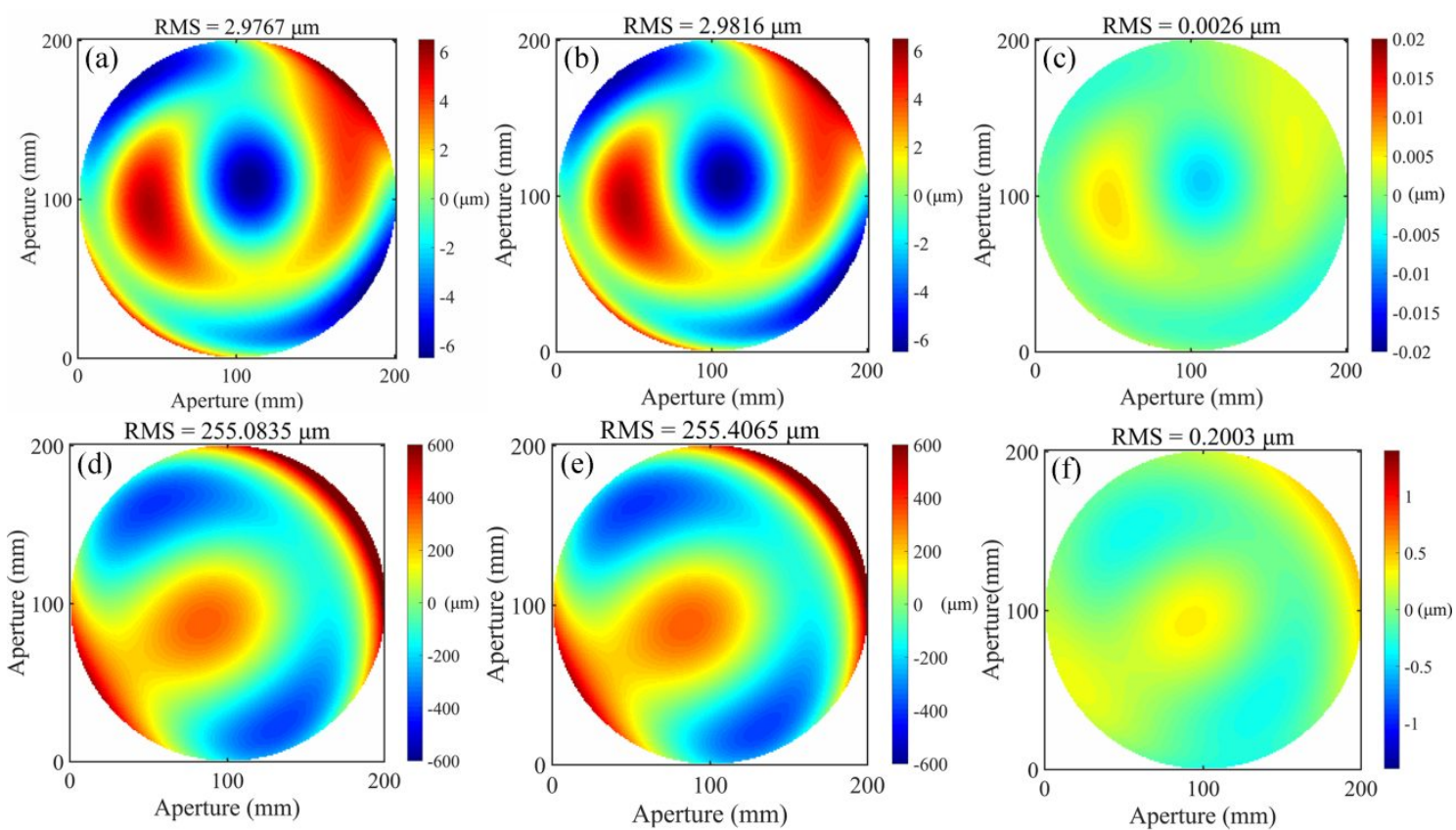

Fig. 3. Surface testing error in simulation, (a) and (d) are actual surface errors of test flat surfaces, (b) and (c) are the measured surface error and the residual error corresponding to (a), (e) and (f) are the measured surface error and the residual error corresponding to $(\mathrm{d})$.

Figures. 3(b) and 3(e) show the surface error results measured by the proposed deflectometric method corresponding to Figs. 3(a) and 3(d), whose RMS value are $2.9816 \mu \mathrm{m}$ and $255.4065 \mu \mathrm{m}$, respectively. Figures 3(c) and 3(f) are the corresponding residual errors between the actual surface errors and measured values with the RMS value of $0.0026 \mu \mathrm{m}$ and $0.2003 \mu \mathrm{m}$. According to Fig. 3, the proposed method provides a feasible way for the testing of refractive surfaces with high dynamic range, in which the residual error for the testing of refractive surface with surface error RMS value of a few microns is in the order of nanometers, and that for the surface with surface error RMS value of hundreds of microns is in the order of submicrons. Thus, accurate testing for refractive surfaces is realized with the proposed testing method based on reverse Hartmann test, with the testing accuracy better than $0.5 \mu \mathrm{m}$. Moreover, the simulation results indicate that the method is not limited to small deformation refractive surfaces, it is applicable for the testing of 
refractive surfaces with large deformation such as industrial refractive surfaces, as long as the surface departure is in the range of deflectometry with high dynamic character.

\subsection{Simulation result of TIR data removal}

To analyze the TIR data and remove it, the simulation of con-centered curved surfaces with $24.675 \mathrm{~mm}$ front surface radius, $22.765 \mathrm{~mm}$ back surface radius and 1.47 refractive index is carried out. A testing system with the same configuration of Fig. 1(b) has been set as a simulation model where there is no tilt and deviation of the surface under test. Figures 4(a) and 4(c) show the spot diagram by ray tracing the testing system, and there are overlapping spots with circle indicator caused by TIR in Fig. 4(a). From Figs 4(b) and 4(d), the surface error with TIR and without TIR can be seen in the test surface.
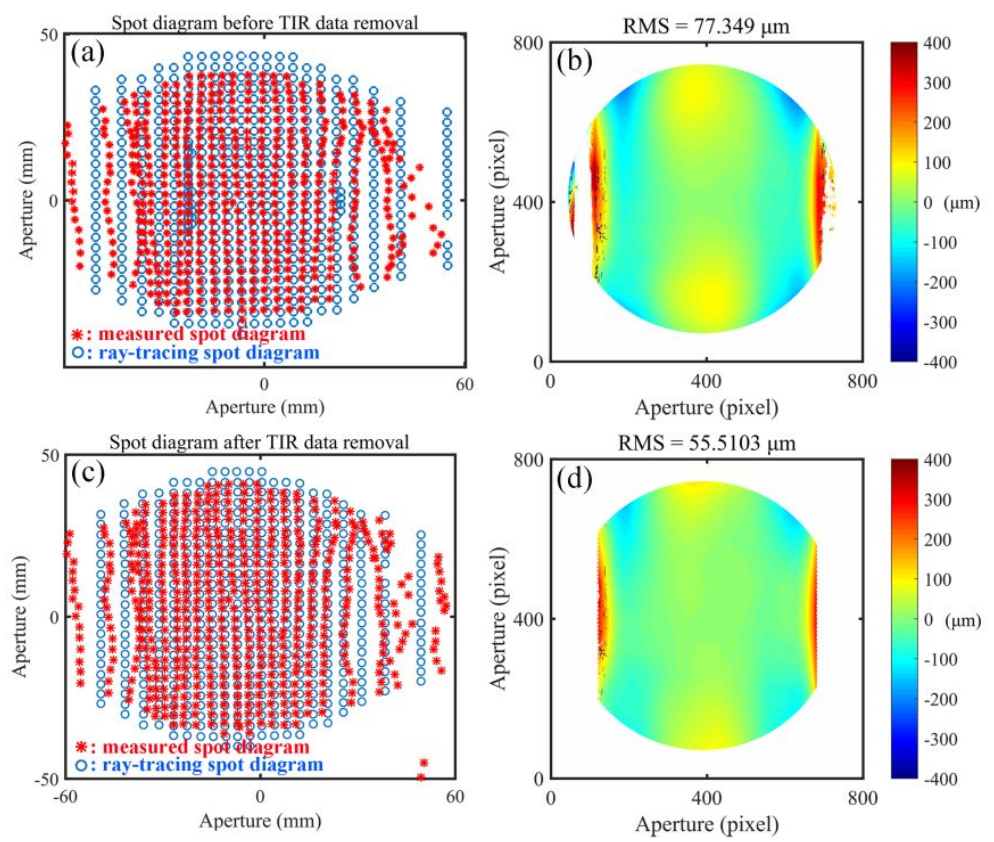

Fig. 4. Measured spot diagram and surface error in two states. Measured spot diagram (a) with TIR data and (c) without TIR data; measured surface error (b) with TIR data and (d) without TIR data.

From the comparison of Figs. 4(a) and 4(c), the overlapping spots with circle indicator caused by TIR have been completely removed in the spot diagram, and the corresponding surface error results are shown in Figs. 4(b) and 4(d). According to Fig. 4, the reduction of impact of TIR has been realized with the surface error RMS value from $77.349 \mu \mathrm{m}$ before TIR data removal to $55.5103 \mu \mathrm{m}$ after TIR data removal.

\section{EXPERIMENT RESULTS}

A deflectometric testing system based on reverse Hartmann test shown in Fig. 1(b) has been set up to demonstrate the feasibility of the proposed method for refractive surface testing. The pixel number of imaging sensor is $1920(\mathrm{H}) \times 1080$ $(\mathrm{V})$. The test refractive object is a cylindrical transparent bottle with $50.86 \mathrm{~mm}$ in external diameter, $2.22 \mathrm{~mm}$ in thickness and 1.51 in refractive index. The system geometry, which includes the exact location of camera, test refractive object and the illuminating screen, are pre-calibrated with three-dimensional machine (Hexagon Metrology Global Classic, accuracy $3.0 \mu \mathrm{m}$ ), the corresponding tilt and the displacement between various parts are then obtained. Based on pre-calibrated system parameter, the testing system is modeled in ray-tracing Zemax software. The acquired illuminating sinusoidal fringes in $x$ and $y$ directions, which are refracted at the test object and captured by camera, are shown in Figs. 5(a) and 5(b). Figures 5(c) and 5(d) are the $x$-slope and $y$-slope data corresponding to fringes in $x$ and $y$ directions, respectively. 

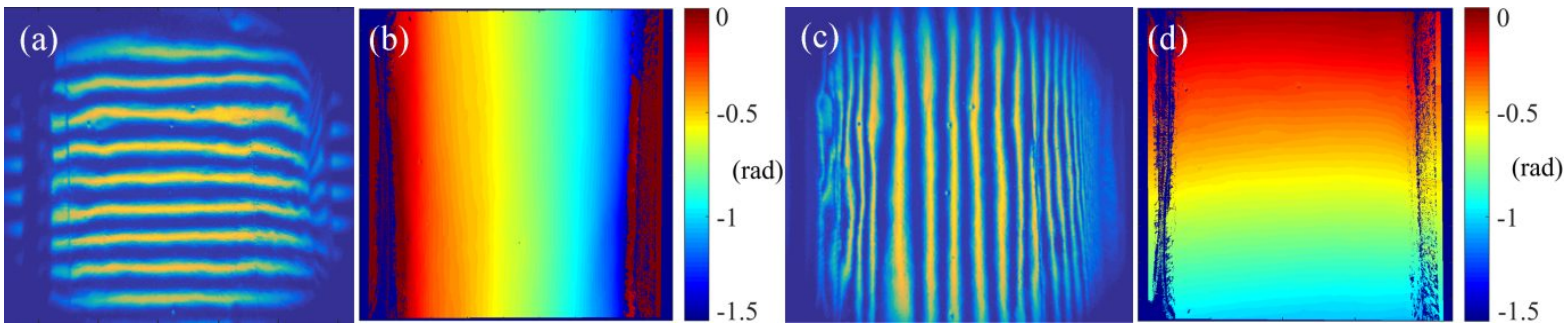

Fig. 5. Acquired sinusoidal fringe and measured slope data in the experiment. Acquired fringes in (a) $x$ direction and (c) $y$ direction refracted by test surface; (b) measured $x$-slope data and (d) measured $y$-slope data.

To evaluate the feasibility of the proposed testing method, repetitive measurements are carried out, in which the test object are placed at three different positions. The test object is removed from Position 1 to Positions 2 and 3 (Table 1), and Figure 6 shows the corrsponding measured surface error results with RMS values of $56.2153 \mu \mathrm{m}, 55.3201 \mu \mathrm{m}$ and $55.0762 \mu \mathrm{m}$, respectively.
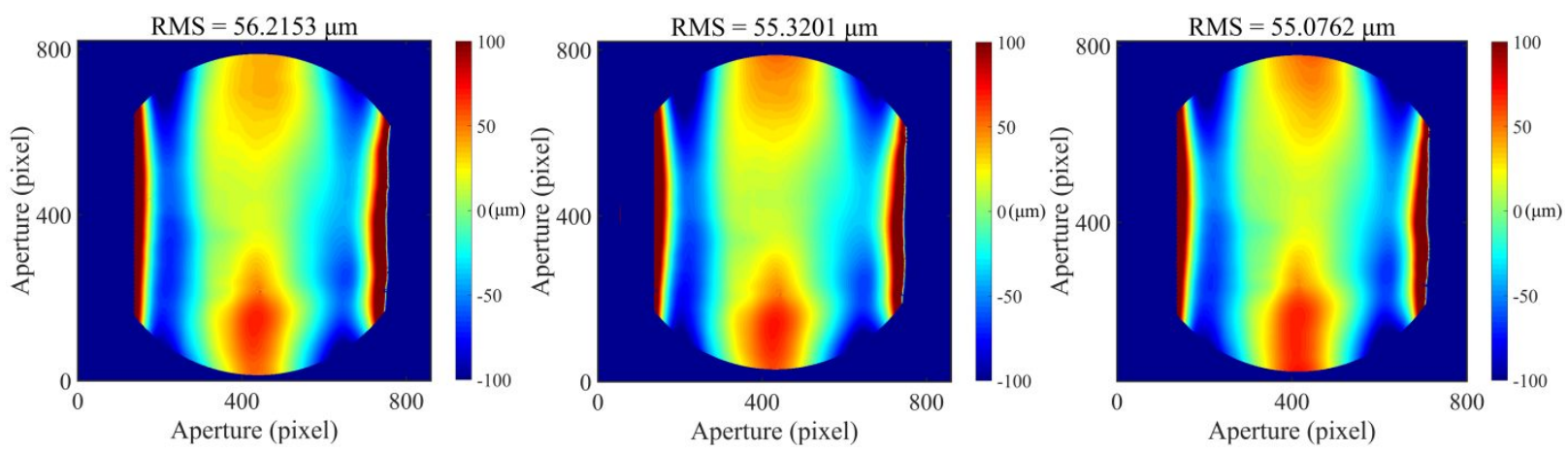

Fig. 6. Measured surface errors in experiment. (a) Measured surface error in Position 1, (b) measured surface error in Position 2, and (c) measured surface error in Position 3.

According to Fig. 6 and Table 1, a good agreement can be seen between the testing results in different positions both in the surface shape and the measured surface error with the RMS difference less than $1 \mu \mathrm{m}$. Thus, good repeatability is achieved in the testing of refractive surfaces with the proposed deflectometric method, and it provides a feasible way to realize the accurate test of refractive surfaces with large deformation.

Table 1. Position and result in experiment results.

\begin{tabular}{|l|l|l|}
\hline \multicolumn{1}{|c|}{ Position } & \multicolumn{1}{|c|}{ Displacement } & \multicolumn{1}{|c|}{ RMS value } \\
\hline Position 1 & $x$ direction: $0 \mathrm{~mm}$ & \multirow{2}{*}{$56.2153 \mu \mathrm{m}$} \\
\cline { 2 - 2 } & $z$ direction: $0 \mathrm{~mm}$ & \multirow{2}{*}{$55.3201 \mu \mathrm{m}$} \\
\hline Position 2 & $x$ direction: $0 \mathrm{~mm}$ & \multirow{2}{*}{$55.0762 \mu \mathrm{m}$} \\
\cline { 2 - 2 } & $z$ direction: $8 \mathrm{~mm}$ & \\
\hline Position 3 & $x$ direction: $-4 \mathrm{~mm}$ & \\
\cline { 2 - 3 } & $z$ direction: $0 \mathrm{~mm}$ & \\
\hline
\end{tabular}

\section{CONCLUSION}

A deflectometric method based on the reverse Hartmann test is proposed for accurate testing of refractive surfaces with large deformation. The virtual "null" test is realized by ray tracing the modeled testing system. With the result of ray tracing and the transverse ray aberration model, the transmitted wavefront from the refractive surface under test is reconstructed. Total internal reflection could exist in edge of curved surfaces, which could introduce significant error in the testing result, and it can be removed based on the mathematical model of the testing system to improve the testing 
accuracy. Both the numerical simulation and experiments have been carried out to demonstrate the accuracy and feasibility of the proposed deflectometric method. A good agreement with testing accuracy better than $0.5 \mu \mathrm{m}$ is realized. The proposed method enables the high-precision measurement of refractive surfaces with large deformation, and it indicates its feasibility for the measurement of industrial refractive surfaces with high dynamic range.

\section{ACKNOWLEDGEMENTS}

The activities of this work are supported by National Natural Science Foundation of China (NSFC) (51375467, 11404312, 51476154, 51404223), Zhejiang Provincial Natural Science Foundation of China (LY17E050014), Zhejiang Key Discipline of Instrument Science and Technology (JL150508, JL150502).

\section{REFERENCES}

[1] Wang, D., Yang, Y., Chen, C. and Zhuo, Y., "Calibration of geometrical systematic error in high-precision spherical surface measurement," Optics Communications 284, 3878-3885 (2011).

[2] Wang, D., Yang, Y., Chen, C. and Zhuo, Y., "Point diffraction interferometer with adjustable fringe contrast for testing spherical surfaces," Applied Optics 50(16), 2342-2348 (2011).

[3] Kammel, S. and Leon, F. P., "Deflectometric measurement of specular surfaces," Transactions on instrumentation and measurement 57(4), 763-769 (2008).

[4] Li, W., Huke, P., Burke, J., Kopylow, C. V. and Bergmann, R. B., "Measuring deformations with deflectometry," Proc. SPIE 9203, 9203F (2014).

[5] Wang, D., Wang, F., Zou, H. and Zhang, B., "Analysis of diffraction wavefront in visible-light point-diffraction interferometer," Applied Optics 52(31), $7602-7608$ (2013).

[6] Wang, D., Chen, X., Xu, Y., Wang, F., Kong, M., Zhao, J. and Zhang, B., "High-NA fiber point-diffraction interferometer for three-dimensional coordinate measurement," Optics Express 22(21), 25550-25559 (2014).

[7] Su, P., Khreishi, M., Huang, R., Su, T. and Burge, J. H., "Precision aspheric optics testing with SCOTS: a deflectometry approach," Proc. SPIE 8788, 87881E (2013).

[8] Knauer, M. C., Kaminski, J. and Hausler, G., "Phase measuring deflectometry: a new approach to measure specular free-form surfaces," Proc. SPIE 5457, 366-376 (2004).

[9] Huang, R., Su, P., Burge, J. H., Huang, L. and Idir, M., "High-accuracy aspheric x-ray mirror metrology using Software Configurable Optical Testing system/deflectometry," Optical Engineering 54(8), 084103 (2015).

[10] Huang, R., Su, P., Horne, T., Brusa, G. and Burge, J.H., "Optical metrology of a large deformable aspherical mirror using software configurable optical testing system," Optical Engineering 53(8), 085106 (2014).

[11]Faber, C., Olesch, E., Krobot, R. and Häusler, G., "Deflectometry challenges interferometry - the competition gets tougher!" Proc. SPIE 8493, 84930R (2012).

[12] Häusler, G., Faber, C., Olesch, E. and Ettl, S., "Deflectometry vs. Interferometry," Proc. SPIE 8788, 87881C (2013).

[13] Wang, D., Zhang, S., Wu, R., Huang, C., Cheng, H. and Liang, R., "Computer-aided high-accuracy testing of reflective surface with reverse Hartmann test," Optics Express 24(17), 19671-19681 (2016).

[14] Su, P., Khreishi, M. A. H., Su, T., Huang, R., Dominguez, M. Z., Maldonado, A., Butel, G., Wang, Y., Parks, R. E. and Burge, J. H., "Aspheric and freeform surfaces metrology with software configurable optical testing system: a computerized reverse Hartamann test," Proc. SPIE 53(3), 031305 (2014).

[15] Huang, R., Su, P., Horne, T., Brusa, G. and Burge, J. H., "Measurement of a large deformable aspherical mirror using SCOTS (Software Configurable Optical Testing system)," Proc. SPIE 8838, 88307 (2013).

[16] Su, P., Parks, R. E., Wang, L., Angel, R. P. and Burge, J. H., "Software configurable optical testing system: a computerized reverse Hartmann test," Applied Optics 49(23), 4404-4412 (2010).

[17] Dominguez, M., Wang, L., Su, P., Parks, R. E. and Burge, J. H., "Software configurable optical test for refractive optics," Proc. SPIE 8011, 80116Q (2011).

[18] Dominguez, M. Z., Armstrong, J., Su, P., Parks, R. E. and Burge, J. H., "SCOTS: a useful tool for specifying and testing optics in slope space," Proc. SPIE 8493, 84931D (2012).

[19] Su, P., Wang, S., Khreishi, M., Wang, Y., Su, T., Zhou, P., Parks, R. E., Law, K., Rascon, M., Zobrist, T., Martin, H. and Burge, J. H., "SCOTS: A reverse Hartmann test with high dynamic range for giant Magellan telescope primary mirror segments," Proc. SPIE 8450, 84500W (2012). 
[20] Su, P., Wang, Y., Burge, J. H., Kaznatcheev, K. and Idir, M., "Non-null full field X-ray mirror metrology using SCOTS: a reflection deflectometry approach," Optics Express 20(11), 12393-12406 (2012).

[21] Rayces, J. L., "Exact relation between wave aberration and ray aberration," Optica Acta: International Journal of Optics 11(2), 85-88 (1964). 